\title{
None More Digital
}

\section{COURTNEY COFFMAN}

Princeton University themselves, like the purposeful use of Adobe Photoshop's checkered transparency background as a material swatch, seen in such works as Curtis Roth's A Provisional Definition of an Architect or T+E+A+M's Ragdale Ring project, Living Picture.

In her recent book, Chromatic Algorithms, author Carolyn Kane describes:

...pure white light continues to carry anachronistic theological associations...its symbolic value runs so deep into the practices of Western, patriarchal, Caucasian culture and its claims to authenticity, origin, innocence, and truth that it has become "natural" to view color as its dirty and degraded counterpart. Where light comes from God and the divine universe, color seeps in from the discarded residue and waste of the fallen, material world. ${ }^{4}$

If color is the detritus of the material world, then there is a codification in the deployment of three specific colors in recent architectural work: pink, blue and black. Perhaps these colors are a contemporary critique, an ideological counter-reaction to the discipline's historical white-washing, as evidenced by Modernism and the Autonomous Project. ${ }^{5}$ Organized into a spectrum of both chromatic value and cultural influence, a soft pink speaks to the politics of taste, a humanist perspective, whereas black introduces a post-humanist future, favoring the mysterious object in its unintelligible depth; a bold blue sits between these binaries as the hue of the technological and the Anthropocene.

If you don't like pink, you don't like yourself.

—Louise Bourgeois

We've reached peak pink. It's been over five years-though probably closer to seven-since pink began to propagate Instagram and the world at large. Much has been written on the cultural impact of a particular soft pink, initially named "Tumblr Pink" but now colloquially known as Millennial Pink-a popular topic from fashion bloggers, to media theorists and even The New York Times, among many others. This specific rosy tint not only represents a moment in which market production finally caught up with the speed of digital media trends, but its emergence during a major shift in identity politics and the retaliation against a dominant paradigm in a movement towards gender-neutrality. As pop-culture's prodigal son, Kanye West, described in 2012: 
Society has put up so many boundaries, so many limitations on what's right and wrong that it's almost impossible to get a pure thought out. It's like a little kid, a little boy, looking at colors, and no one told him what colors are good...Why would anyone pick blue over pink? Pink is obviously a better color. Everyone is born confident, and everything's taken away from you. ${ }^{6}$

It's hard to deny what was once a taboo color has now saturated our daily environment-is there ever a day without pink? It can be seen everywhere online and in real life: architecture, art exhibitions, animations, fashion, food, graphic design, makeup, music, sex toys, tech devices, wine-even Log updated its cover to muted pink for issue 41 through 44 . It is no wonder this color has gained major momentum in current architectural projects and representation given that practices continually facilitate social media platforms as a tool for discourse. This digital proliferation also happens to parallel the resurgence of post-modern pastiche, in a satirical adaptation of soft color palettes from the 1980s and early 90s. This pink, however, doesn't shy away from description of cute, pretty or feminine; rather it embraces and bolsters these qualities if not to exemplify them, then at least to juxtapose them with the sophistication of a digital finesse. As it circulates through countless reposts and hashtags, pink has unleashed multiple readings of fluid meaning, but its overuse in commercial applications has diluted pink into a cliché. To mediate this moment, the following projects demonstrate the multi-valent nature of this digital phenomenon with critical thought.

The Kid Gets Out of the Picture, a collaborative installation at Materials \& Applications in 2016 with The LADG, First Office, Hirsuta, Laurel Broughton and Andrew Kovacs, presented a "contemporary update on the aesthetic principles of early 19th century English landscape architecture." To describe this new aesthetic is a challenge-it oscillates between everyday construction materials and complex digital geometries. But the most striking feature of this landscape are the gently rolling, pink plaster lumps. ${ }^{7}$ The fleshy surfaces encouraged visitors to sojourn across the installation to its peak, look out over Los Angeles' Silver Lake Boulevard below, and take in the sublime views of the foothills littered with houses. In the choreographed performance, From Above | Looking Down, five dancers maneuvered around and below the installation deploying, "a series of improvised scores that explore how to move with and through comfort." Fully dressed in a matching pink athleisure, the dancers softly blended into the landscape, disappearing into its folds, cradled by its small valleys. "Drawing from the performers' personal histories with their dance and movement practices and our political present," Choreographer Gwyneth Shanks describes, "the piece shifts between the kinetic and the metaphoric, the individual and the collective, searching for ways to offer and receive comfort, care, and support." ${ }^{8}$ The resulting performance was feminine, vulnerable, human-in other words, it was truly pink.
Sympathy is a bi-product of pink, as witnessed by T+E+A+M's 2016 Detroit Reassembly Plant, which proposes an alternative approach to rehabilitative projects. Their proposal recognizes "architecture's capacity to work on and produce both materials and images" and "aims to reverse current perceptions of Detroit." In an effort to avoid propagating yet another ruin porn image of Detroit, $\mathrm{T}+\mathrm{E}+\mathrm{A}+\mathrm{M}$ reframes Detroit's derelict materiality through rose-colored lenses. ${ }^{9}$

In the cultural imaginary of Detroit, vacant properties and their debris are synonymous with neglect, crime, economic hardship, and the fallout from the city's postindustrial decline. Our project ascribes value to this building detritus and trans-forms Detroit's scarcity of resources into an abundance.

A project about optimism and optimization, piles of seemingly crude, raw materials - the remnants of buildings past-are backdropped by a soft pink glow. The color sneaks into the monochromatic models under the building mass, in the shadows and injecting excitement through-out the project drawings. Again, consider Kane's point on how color was historically viewed as ungodly, "the discarded residue and waste of the fallen, material world," a description that strongly correlates color with a city abandoned by a failed market.

Described as "a travelogue tracing the online journey of nine architectural instruments, from renderings produced for the pleasure of airborne algorithms to digital models negotiating the online bureaucracies of copyright legislation," Curtis Roth's 2017 publication, Some Dark Products, is a brilliant, post-capitalist take on the bizarre practices of a global market in the digital age. Forget about pink of the Acne Studios shopping bag, the Glossier makeup set, the rose-gold Apple products, or the Rosé-all-day vibes, Roth's use of pink inverts the market appeal of Millennial Pink to reveal the hidden production and labor systems of architecture in the internet age. ${ }^{10}$ It's quite impossible to understand what comprises the pink digital artifact behind the page text, but much like the books content, it suggests an underlying system at work behind the scenes of what is legible to our eye. Occasionally, the grainy pink noise interrupts the images, breaking the edges of bounding boxes and page spreads. It's hard to know if the book is a screen capture, or if there is background task continually running as the pages turn. Shattering a conceptual fourth wall of the digital screen, this virtual space bleeds out into the physical space of the publication, further conflating the pink of reality and online.

...each painting's blue world, although of the same blue and treated in the same way, revealed itself to be of entirely different essence and atmosphere... ${ }^{11}$

-Yves Klein 
Yves Klein installed ten monochromatic paintings for ten days in Milan. ${ }^{12}$ The canvases debuted his International Klein Blue (IKB), a conception of "pure idea" to patent. ${ }^{13}$ Each painting was equally identical in dimension and orientation, but mounted at different heights away from the gallery walls and variously priced-a critique on ways of 'seeing' art, the status of the art object, and the market. This avant-garde moment further obfuscates in later years as IKB assumes other multimedias forms, each with their own art and economic value. But as we move away from the physical pigments of IKB, questions of aura and authenticity emerge. In his 1993 work, "Blue," Derek Jarman documents a photograph of Klein's monochrome painting "IKB 79" on 35mm film. The audience is conceptually catapulted deep into IKB through the painting itself, a media of suspended pigments in resin; the photographed image, a fix of suspended of silver and dye couplers; the $35 \mathrm{~mm}$ film, a plastic emulation of silver salts and gelatin. The media of the $35 \mathrm{~mm}$ reel is not that far removed from the material composition of the first IKB paintings; perhaps the 1957 show was a multi-media exhibition, a room of primitive screens. Pushing the abstraction of color further, consider the translation of "Blue" when the Museum of Modern Art acquired Jarman's work into their permanent collection, required the digitization of the analog. And it is here where the recent surge of blue is situated: from the auxiliary channels on televisions to the blue-screens used in film productions for post-production digital effects. ${ }^{14}$

This is the blue of the Anthropocene; it synthesizes, simulates and expedites natural objects that have been acted upon by human systems. In 2017, Adrian Villar Rojas swathed the MoCA Geffin Chroma-key Blue for his third iteration of "The Theater of Disappearance." The massive exhibition space conceptually performed as a blue-screen set littered with the props of real rocks and the fake archaeologies of postconsumer waste. The exhibition flattens ontologies through its constructed fictional stories with everyday objects-the reality of impermanence superimposed by preservation. These crude objects-sedimented resin totems, large rocks, and large refrigerated cases-were dimly lit by the cool fluorescent lights of industrial kitchen equipment. ${ }^{15}$ Playing the role of post-capitalist explorer, visitors were advised by docents to use the flashlights on their handheld black boxes to navigate the foreign terrain. A towering series of parallel blue walls suggest the existence of multiple realities, simultaneously collating space and compressing time, much like the familiar digital space of numerous internet browser windows opened all at once.

Laida Aguirre also commandeered the blue-screen set and created an environment for the invisible political economies of shipping in her 2018 project Careful Crates. An exploration in the culture of logistics, the research focused on the architectural impact of shipping ecologies, moving between the human scale-such as obtaining a package signature-to the global scale of data management. In this context, it's interesting to consider YInMn Blue, a highly saturated pigment somewhere between a Cobalt Blue and International Klein Blue. Mas Subramanian, Professor of Materials Science at Oregon State University, discovered this new blue-the first in over 200 years-when he attempted to create a material compound conductive for high temperatures in electronics. He stumbled upon an unknown pigment, a compound of yttrium, indium and manganese oxide colloquially known as YInMn Blue, which maintains functional chemical properties as a stable material to deflect heat. Ultimately, this blue makes data travel at a faster rate. Just imagine the possibilities of YInMn Blue in the workflow of Careful Crates...

Other current examples of blues in architecture include the Becoming Digital conference at The University of Michigan, "a year-long project that considers the deep changes underway in architecture and visual culture caused by the increasing ubiquity and naturalization of digital technology." ${ }^{16}$ Interestingly enough, a blue, cross-hair grid proliferates across the event collateral, reinforcing this intense color as explicitly linked to post-digital discourse. A scaleless monument to misalignments, Viola Ago and Hans Tursack's "Thick Skin" also dons the blue. This future relic, demonstrates architecture's current fascination with misreading form through applied graphics to achieve a complex object outside of digital space. And, most recently, "The Mile-Long Opera: a biography of 7 o'clock" on The Highline in New York-co-created by Diller Scofidio + Renfro and composer David Lang. Between West 15th and West 16th Street, audience members were submerged into a blue tunnel while the Huaxia Choir and MLO Company Singers serenaded from their individual mini stages. This moment can be experienced through the event website, with features like "Walk the Mile in 360," where for three-minutes and 57 seconds - or 353 feet of The Highline-virtual visitors can witness the choral sounds of "makeshift," No. 6 in the 26-part arrangement. The experiential happening dissolves into digital ephemera, or the immaterial-circumventing Klein's blue once again.

It's like, how much more black could this be? And the answer is none. None more black.

\section{-Nigel Tufnel in This is Spinal Tap}

Black is the penultimate. It is the color beyond the Anthropocene, the shade of a post-human age-and all those other hyperobject(ified) buzzwords currently swirling about in the atmo(blogo)sphere these days. The depth of black is performative, as Nigel Tufnel demonstrates when deliberating with bandmates David and Derek, along with manager lan over their "Smell the Glove" album cover in This is Spinal Tap, a mock-rockumentary: 
Derek: You can see yourself in... both sides.

David: I feel so bad about this...

Nigel: It's like a black mirror.

David: Well, I think it looks like death...it looks like mourning. I mean it looks...

lan: David, every movie, in every cinema is about death; death sells!

Nigel: I think he's right, there is something about this, that's so black, it's like; "How much more black could this be?" and the answer is: "None, none...more black."

David: I think, like you're rationalizing this whole thing into something you did on purpose. I think we're stuck with a very stupid and a very dismal looking album, this is depressing.

Nigel: David, it's a choice.

Black's mystery-its allure-creates an opportunity for multiple (mis)readings and (mis)interpretations. Think back to the purposefully obscure architectural renderings of Hugh Ferriss, and the depth of architectural mass through simple charcoal shading. "From being a helper, the great delineator becomes a leader," Rem Koolhaas describes Ferris and the power of his dark abstractions in the development of Manhattan's early 20th-century towers. According to Koolhaas, Ferris' most impactful contribution to the city is "the creation of an illuminated night inside a cosmic container, the murky Ferrissian Void: a pitch black architectural womb that gives birth to the consecutive stages of the Skyscraper...and promises to generate ever-new ones." 17 It is in this conceptual space of abstraction where contemporary black projects are situated; the generative inner-worlds and sublime cosmic connections are massive undertakings for architecture.

In Tom Wiscombe's 2015 Guggenheim Helsinki entry, he proposes a black crystalline form. As the dark object floats in space, an intentional removal from the ground plane both physically and metaphorically elevating the museum, it creates a world-in-itself. The project expresses, "vicarious relations to the local context of Helsinki, while simultaneously withdrawing...to create a discrete and internalized world for art. This withdrawal is intended to incite and confound speculation on the building's origins, to further create a mysterious sense of depth beyond superficial qualities." ${ }^{18}$ Within the thick poché of the museum's scale-less form are a pair of secondary volumes containing galleries. These nested forms also don a layer of poché, a generative form-making technique of black in architectural representation cleverly rendered in real space and time. "Circulating through the museum, visitors find themselves momentarily suspended above a deep, almost geological chasm. Rather than experiencing a fluid continuum of art, visitors must leap between worlds, culminating in a sense of there being no ground." These interior worlds are hidden from view, purposefully illegible on the museum's exterior and further obfuscated by the black composite façade.

Illegibility is a common characteristic of this contemporary black architecture; it is theoretically described as the ambivalent object. As Jason Payne highlights, "the base condition of uncertainty is one thing, but the conscious awareness of this state of being is something more: ambivalence. To be ambivalent is to choose to be unclear, undecided, and equivocal." ${ }^{19}$ In the aforementioned 2017 installation, The Kid Gets Out of the Picture, Payne designed two decontextualized rocks-Ida and Dactyl, an asteroid and its moon-for the pink picturesque landscape. Through these obscure black objects, Payne proposed a deeper registration in the marginal percentage of dark tonality in William Gilpin's 18th-century drawings. Initially painted in the Gilpin palette, Ida was a fabricated in pastel colors then completely masked by a slick, black resin. "As a study in black more generally this object has no meaningful relationship to the surrounding landscape of other objects it sits among on exhibition. At the same time its various blacks are properly picturesque and so in theory it should fit in." Ida denies the picturesque ideal and its pastel past through primitive form and layers of deep black. ${ }^{20}$

The prevalent use of black on architectural objects means a loss in resolution and detail, which makes reading them all the more difficult, like the dark side of the moon. Perhaps no other black expresses this difficulty more than Surrey NanoSystems' Vantablack-defined as a material and not a color. ${ }^{21}$ Absorbing $99.96 \%$ of light, this black makes a solid void and renders space obsolete as three-dimensions are flattened into two-dimensions. Vantablack is the complete absence of color, it is none more black and the closest experience to a blackhole humans may ever experience.

It is the most resistant and antithetical to existing color systems, historical treatises, our own biological optic comprehension, and perhaps even to our existing digital tools-how does one design with this black? It's interesting to consider that the ambivalent architectural object-and the digitally oriented architect-would seek the same effects of Vantablack, ultimately returning the us back to the material of pigments.

It is none more black and perhaps none more digital.

\section{ENDNOTES}

1. In reference to the conference panel prompt: "To become digital is to exist in a digital world. It is an ontological state that tacitly recognizes pervasive technology, computational logic, and digital aesthetics as the background condition to everyday life. To become digital is to be situated in a context where everything from screen to stone exists as data and matter, where habits of mind forged within the digital environment are constantly transferred to the analog world." 
2. The Bauhaus played an integral role to the integration of color theory courses in design curriculums. It was Bauhaus student Josef Albers who elevated color not merely as theory or practical thought but as a practice that must be experienced, particularly through making. Albers' students experimented and theorized color and composition in his course at Black Mountain College.

3. A reference to Jacqueline Lichtenstein's Couleur bloquente: Rhitorique et peinture a l'age Classique by Sylvia Lavin regarding the "conflict over whether painting should be organized around meaning or affect." Sylvia Lavin, "What Color is it Now?" Perspecta 35 (2004): 102-103. This research is indebted to this particular essay by Lavin.

4. Kane's book makes for an excellent guidebook to navigating color theory in a digital world. Carolyn Kane, Chromatic Algorithms: Synthetic Color, Computer Art, and Aesthetics after Code (Chicago: University of Chicago Press, 2014).

5. In her recent academic lectures, Jennifer Bonner has proposed a new movement of emerging practices, The Pinks-the generation proceeding The Whites and The Grays.

6. Kanye West interviewed by Peter Macia, "Kanye West: I'm Amazing," The Fader 58 (November 2012).

7. In conversation with The LADG's co-founder, Ben Freyinger, on the use of pink in The Kid Gets Out of the Picture, his response diverted to a previous conversation with Neil Denari, who also inquired about the use of pastel colors in The LADG's recent work, along with other emerging practices, to which Freyinger replied, "The generation of architects before us used up all the good [crayon] colors and we are stuck with the shitty pastels no one wants in the box."

8. "From Above / Looking Down" as conceived by Gwyneth Shanks.

9. $T+E+A+M$ 's elevator pitch: "Detroit doesn't have a material problem; its material has an image problem."

10. Roth further elaborates, "In a world where the physical processes of architecture are becoming increasingly less important and digital processes proliferate through all phases of architectural ideas and documentation, we should perhaps be looking to understand the ways in which architects work, and examine how we can claim the processes-not just the products-of our labors."

11. "All of these blue proposition, all alike in appearance, were recognized by the public as quite different from one another....The most sensational observation was that of the "buyer." Each selected out of the pictures...pictures the one that was his, and each paid the asking price. The prices were all different of course." Yves Klein in Benjamin Buchloh's "Andy Warhol's One-Dimensional Art: 1956-1966," in Andy Warhol, October Files (Cambridge, MA: The MIT Press, 2001), 31.

12. "Yves Klein: Proposte monocrome, epoca blu" exhibited at the Galleria Apollinaire in Milan, Italy, January 2-12, 1957.

13. Developed with the paint expertise of Edouard Adam, the ultramarine pigment was suspended in Rhodopas, a synthetic resin, which was later patented by Klein, to maintain authenticity of "the pure idea."

14. Green screen is usually preferred because its farthest away from human skin tones; since green is most prevalent in nature, blue is the preferred alternative. This is an interesting concept when considering architectural objects outside of paper space and placed into the environment of 'The Real' world.

15. Within these refrigerated cases was a mélange of natural materials-including foods, such as cakes, cheese, grapes, fish-along with synthetic, man-made products, like sneakers, pipes, and plastic-coated cables. Suggestive of Dutch still life paintings, the cases were colorful vignettes that slowly turned grotesque as the natural systems of mold and rot overtook the organic materials: subsequently, the synthetic materials were the most vibrantly colored object remaining in the cases by the end of the exhibition.

16. According to their Instagram account @becommingdigital.

17. See Rem Koolhaas, Delirious New York (New York: The Monacelli Press, 1994), 110-117.

18. Visit https://tomwiscombe.com/GUGGENHEIM-HELSINKI for full project description.

19. Over the last several years, Payne lead multiple tech-seminars at UCLA centering on the ambivalent object; from disco balls to asteroids to bunkers, there are a multitude of tangible objects with uncanny, otherworldly qualities ripe for architectural experimentation. The success of representing these ambivalent objects often relies on the correct selection of materials, such as printer paper and proper ink, among other dark-colored materials. Payne's tech-seminars required highly controlled rule sets and outputs, perhaps in an attempt to eliminate the human (hand) altogether. Except from Jason Payne, "Variations on the Disco Ball, or The Ambivalent Object," Project Journal 2 (2013): 20-27.

20. "We often work with asteroids in our projects for their ease of translation to otherworldly primitives. If there were the possibility to select 'Primitive: Other' in our modeling software then surely the result would always be asteroids both for their proximity to and distance from the ideal forms." Jason Payne, "Ida (and Dactyl): A Darker Register of the Picturesque," Offramp 12 (Fall/Winter 2016). https://offramp.sciarc.edu/articles/ ida-and-dactyl-a-darker-register-of-the-picturesque.

21. Comprised of carbon nanotubes according to Surrey NanoSystems. 